\title{
Routine Chemoprophylaxis for Venous Thromboembolism in Orthopedic Patients: Is it Justified?
}

\author{
Prasoon Kumar ${ }^{1}$, Mandeep S Dhillon ${ }^{2}$, Rohit Kansal ${ }^{3}$
}

\begin{abstract}
Introduction: Orthopedic patients require prolonged immobilization and undergo major surgical procedures which make them susceptible to venous thromboembolism (VTE). The role of chemoprophylaxis to prevent VTE is still debated, with many surgeons using it routinely, while certain centers use it sporadically. The present review assesses the evidence in literature regarding the VTE chemoprophylaxis in different subsets of orthopedic patients with specific focus on arthroplasty, pelvic trauma, and long-bone fractures.

Materials and methods: A PubMed search to look for current usage of routine chemoprophylaxis in hip/knee arthroplasties and postoperative cases of pelvic-acetabular, hip, and tibia fractures yielded 2,216 hits. Based on the relevant articles, the evidence in literature was studied to determine the role of chemoprophylaxis.

Results: The incidence of VTE in orthopedics patients is well documented with several studies, both Indian and Western quoting rates of up to $72 \%$. There are no guidelines from any Indian orthopedics body or society, and most of the surgeons rely on the recommendations from the American Academy of Orthopaedic Surgeons (AAOS) and the American College of Chest Physicians (ACCP). These guidelines are based on the review of the literature and recommend routine chemoprophylaxis to prevent VTE in hip/knee arthroplasties and hip fractures. Low molecular weight heparin, apixaban, rivaroxaban, and fondaparinux are potential agents. Oral low-dose aspirin is also effective in arthroplasty patients, while its efficacy may not be at par with the other agents in trauma cases; the evidence is however still not clear. There is no similar role of routine chemoprophylaxis in fractures distal to the knee; as the thrombi formed are distal, they do not often embolize to the lungs and are rarely clinically significant.
\end{abstract}

Conclusion: Patients undergoing hip and knee arthroplasties or surgeries for pelvic-acetabular and hip fractures require routine VTE chemoprophylaxis. LMWH or newer agents like apixaban or fondaparinux are effective in decreasing the incidence of clinical VTE and could be prescribed for a period as long as 6-8 weeks postoperatively.

Keywords: Acetabulum fractures, Arthroplasty, Chemoprophylaxis, Deep vein thrombosis, Long-bone fractures, Pelvic fractures, Pulmonary embolism, Venous thromboembolism.

Journal of Postgraduate Medicine Education and Research (2019): 10.5005/jp-journals-10028-1334

\section{BACKGROUND}

Venous thromboembolism encompasses both pulmonary embolism and deep vein thrombosis (DVT). ${ }^{1}$ The latter could be a fatal entity as more often than not it is a silent phenomenon without clinical signs, and mortality could be the first manifestation. VTE is a frequent complication in orthopedic patients undergoing hip or knee arthroplasties, and with pelvic-acetabular and long-bone fractures. However, the incidence rates vary in literature (0-72\%) and practice of routine chemoprophylaxis in these patients is not standardized. ${ }^{2-4}$ An early study by Nagi et al. reported an incidence of $8 \%$ proximal DVT after major lower limb surgeries over a 14 months period; they recommended routine prophylaxis after such surgeries. ${ }^{5}$ Agarwala et al. in their 104 patients reported $34.4 \%$ incidence of DVT with prophylaxis and $60 \%$ without it. Patients undergoing total knee replacements were found to be at the highest risk. ${ }^{2}$ Sen et al. in their review reported $8 \%$ incidence of VTE in trauma patients receiving chemoprophylaxis and $14.5 \%$ in those without the prophylaxis. ${ }^{6}$ They recommended routine prophylaxis in trauma patients. However, Bagaria et al. and Mavalankar et al. recommend prophylaxis only in those patients of hip and lower limb fractures who are at high risk owing to other coexisting factors. ${ }^{7,8}$ Herein lies the controversy as even today many orthopedic surgeons are divided about routine use of chemoprophylaxis.

We reviewed the literature and assess the current trends and available evidence for routine chemoprophylaxis in major subsets of orthopedic patients who develop VTE.

\begin{abstract}
${ }^{1-3}$ Department of Orthopaedics, Postgraduate Institute of Medical Education and Research, Chandigarh, India

Corresponding Author: Mandeep S Dhillon, Department of Orthopaedics, Postgraduate Institute of Medical Education and Research, Chandigarh, India, Phone: +91 9815951090, e-mail: drdhillon@gmail.com

How to cite this article: Kumar P, Dhillon MS, Kansal R. Routine Chemoprophylaxis for Venous Thromboembolism in Orthopedic Patients: Is it Justified? J Postgrad Med Edu Res 2019;53(4):152-157.
\end{abstract}

Source of support: Nil

Conflict of interest: None

\section{Materials and Methods}

A PubMed search was done on 3rd February 2019 to look for the current usage of routine chemoprophylaxis for VTE in three subsets of orthopedic patients. The first search was for arthroplasty patients with keywords "venous thromboembolism"(MeSH Terms] OR ("venous"[All Fields] AND "thromboembolism"[All Fields]) OR "venous thromboembolism"[All Fields]) AND ("arthroplasty"[MeSH Terms] OR "arthroplasty"[All Fields]) AND ("prevention and control"[Subheading] OR ("prevention"[All Fields] AND "control"[All Fields]) OR "prevention and control"[All Fields] OR "prophylaxis"[All Fields]). This yielded 1578 hits.

The second search for cases of pelvic-acetabular and hip fractures with keywords ("venous thromboembolism"[MeSH Terms] 
OR ("venous"[All Fields] AND "thromboembolism"[All Fields]) OR "venous thromboembolism"[All Fields]) AND ("prevention and control"[Subheading] OR ("prevention"[All Fields] AND "control"[All Fields]) OR "prevention and control"[All Fields] OR "prophylaxis"[All Fields]) AND ("pelvis"[MeSH Terms] OR "pelvis"[All Fields] OR "pelvic"[All Fields]) AND ("hip fractures"[MeSH Terms] OR ("hip"[All Fields] AND "fractures"[All Fields]) OR "hip fractures"[All Fields] OR ("hip"[All Fields] AND "fracture"[All Fields]) OR "hip fracture"[All Fields]) yielded 384 hits.

A third search focused on tibia fractures with keywords ("venous thromboembolism"[MeSH Terms] OR ("venous"[All Fields] AND "thromboembolism"[All Fields]) OR "venous thromboembolism"[All Fields]) AND ("prevention and control"[Subheading] OR ("prevention"[All Fields] AND "control"[All Fields]) OR "prevention and control"[All Fields] OR "prophylaxis"[All Fields]) AND ("tibial fractures"[MeSH Terms] OR ("tibial"[All Fields] AND "fractures"[All Fields]) OR "tibial fractures"[All Fields] OR ("tibia"[All Fields] AND "fracture"[All Fields]) OR "tibia fracture"[All Fields]) yielded 254 hits.

Relevant articles studying the role of chemoprophylaxis in all three orthopedic situations of arthroplasty, pelvic-acetabular, hip, and tibia fractures were screened and studied to determine the evidence in literature.

\section{Hip/Knee Arthroplasty}

VTE is a known complication associated with hip and knee replacements. Different pros and cons of routine prophylaxis need to be looked at before initiating chemoprophylaxis, as this can also cause postoperative bleeds or wound hematoma. ${ }^{9}$ Persistent discharge from the surgical site leading to infections can negate the desired outcomes. Currently, there are no clearcut recommendations for chemoprophylaxis focusing on Indian patients. Most orthopedic surgeons use the American Academy of Orthopedic Surgeons (AAOS) recommendations for VTE in arthroplasty patients. ${ }^{10}$ Earlier guidelines in literature were debatable as the safety of the regimen was overlooked. ${ }^{10,11}$ The role of low-dose aspirin was somewhat controversial and AAOS recommendations incorporated all these concerns in 2011. There are ten recommendations in grades of strong, moderate, weak, inconclusive, or consensus. There were one strong and three moderate recommendations while the majority of the remaining ones were based on consensus (Table 1).

The guidelines state that routine screening of these patients is not necessary (strong) and preoperatively antiplatelet drugs should be discontinued (moderate); however, Lieberman and Pensak ${ }^{9}$ observe that in the subset of arthroplasty patients, data are scarce regarding the second point. The stoppage of aspirin before surgery is controversial, and if there is a pro-thrombotic risk due to cardiac issues, it may still be continued. ${ }^{12}$ Similarly, stopping clopidogrel prescribed after cardiac stents may cause increased thrombosis on stoppage. The current recommendation for orthopedic procedures is stoppage of clopidogrel 1 week before surgery, while aspirin does not require stoppage. ${ }^{12}$

On the contrary, the AAOS recommendations do not mention specific agents or duration of prophylaxis and it is left for the surgeons to decide in individual situations. ${ }^{10}$ One recommendation based on consensus vaguely states that patients with bleeding disorders may not be given chemoprophylaxis. This will need routine screening of all bleeding disorders in every patient, which is certainly an overkill. Adequate history and clinical records may help in this regard. AAOS also advises only mechanical prophylaxis in patients with liver diseases and use of neuraxial (spinal/epidural) anesthesia as it lowers the blood loss, probably owing to the induced hypotension. ${ }^{10}$

The American College of Chest Physicians (ACCP) (in their older version) suggested that aspirin has no role in prophylaxis. Initially, their recommendations were based on end results, which even included asymptomatic VTE diagnosed with venography. ${ }^{13}$ These controversial points were modified in the latest guidelines, where focus is shifted toward reduction in clinically significant VTE. ${ }^{14}$

Their guidelines include grading of the recommendations as 1 or 2 based on a systematic review of literature; grade I implies that the efficacy of prophylaxis certainly outweighs the risks based on concrete evidence, whereas grade II implies that the level of available evidence is low quality and uncertainty persists regarding the pros vs cons of the prophylactic agents. ${ }^{14}$ The quality of the included studies was assessed with three subgradings of $A, B$, and $C$ where $C$ is based on observational studies only. These guidelines also advise against routine screening for VTE similar to AAOS. The ACCP recommends either chemoprophylaxis or mechanical devices for a minimum of 2 weeks. No patient should be without prophylaxis. Even aspirin has been reported to be equally efficacious to other agents like low molecular weight heparin (LMWH), fondaparinux, apixaban, and rivaroxaban for this purpose. ${ }^{14}$ This was on the basis of pulmonary embolism prevention (PEP) trial which concluded that aspirin with its oral dosage and nonrequirement of any sort of monitoring is an ideal agent for chemoprophylaxis after hip or knee arthroplasty as well as surgeries for hip fractures. ${ }^{15}$ Administration of $L M W H$ at $\geq 12$ hours prior to or postsurgery is recommended. Regarding the overall duration of therapy, up to 35 days of prophylaxis is advised; however, the evidence is $2 \mathrm{~B}$ and of low quality.

To summarize the key points from these two guidelines, we have enough evidence supporting chemoprophylaxis after hip and knee arthroplasties for a period up to 6 weeks postsurgery. Mechanical devices can be used as add-ons to supplement the drugs. LMWH like clexane and apixaban are the commonest drugs used. Aspirin as the sole agent is much better than no prophylaxis at all and may be prescribed. At our institute, we use tablet apixaban (Eliquis) 2.5 milligram twice a day from postoperative day 2, 12 hours after the epidural catheter is removed, for a period of 4-6 weeks. In total knee replacements, we additionally prescribe mechanical compression stockings.

\section{Trauma as a Risk Factor}

Unlike elective surgeries like arthroplasties, pathogenesis of VTE in trauma starts immediately after injury, much before the patient presents to the hospital. The formation of thrombin starts within 24 hours of trauma, and the procoagulation factors can remain increased for up to 14 days. ${ }^{16}$ In addition, dehydration, immobilization, and transfusions of blood products add to the hypercoagulable state. ${ }^{17,18} \mathrm{Up}$ to $68 \%$ of thrombi associated with trauma are proximal, which have a higher chance of proximal propagation and embolization than distally situated thrombus. ${ }^{17,19}$ Most of the trauma cases need some kind of surgical intervention, minor or major, which may add to further insult and can result in dislodgement of preformed thrombus, thereby increasing chances of embolic events. ${ }^{18-20}$ Thus, the role of chemoprophylaxis becomes important, especially in fractures of pelvic-acetabulum and longbone fractures like femur and tibia. 
Table 1: AAOS guidelines

\begin{tabular}{|c|c|}
\hline No. & AAOS recommendations \\
\hline 1 & $\begin{array}{l}\text { Routine postoperative duplex ultrasonography screening of patients who undergo elective hip or knee arthroplasty is not } \\
\text { recommended }^{\text {a }}\end{array}$ \\
\hline 2 & $\begin{array}{l}\text { Patients undergoing hip/knee arthroplasty are already at high risk of venous thromboembolism. The practitioner might further } \\
\text { assess the further risk of venous thromboembolism by determining whether these patients had any previous history of VTE }\end{array}$ \\
\hline 3 & $\begin{array}{l}\text { Patients undergoing elective hip/knee arthroplasty are at risk of bleeding and subsequent complications. Therefore, these patients } \\
\text { must be assessed for known bleeding disorders like hemophilia and for the presence of any active liver disease which further } \\
\text { increase the risk of bleeding and its complications }\end{array}$ \\
\hline 4 & Antiplatelet agents (e.g., aspirin, clopidogrel) before undergoing elective hip or knee arthroplasty may be discontinued ${ }^{b}$ \\
\hline \multirow[t]{3}{*}{5} & $\begin{array}{l}\text { Suggest the use of pharmacologic agents and/or mechanical compressive devices for the prevention of venous thromboembo- } \\
\text { lism in patients undergoing elective hip or knee arthroplasty, and who are not at elevated risk beyond that of the surgery itself for } \\
\text { venous thromboembolism or bleeding }\end{array}$ \\
\hline & $\begin{array}{l}\text { There is no ample evidence about which prophylactic strategy (or strategies) is/are optimal or suboptimal. Therefore, no } \\
\text { recommendation for or against specific prophylactics in these patients are given }{ }^{d}\end{array}$ \\
\hline & $\begin{array}{l}\text { In the absence of reliable evidence about how long to employ these prophylactic strategies, it is the opinion of this work group that } \\
\text { patients and physicians discuss the duration of prophylaxis }{ }^{\mathrm{e}}\end{array}$ \\
\hline 6 & $\begin{array}{l}\text { In the absence of reliable evidence, it is the opinion of this work group that patients undergoing elective hip or knee arthroplasty, } \\
\text { and who have also had a previous venous thromboembolism, receive pharmacologic prophylaxis and mechanical compressive } \\
\text { devices }^{\text {e }}\end{array}$ \\
\hline 7 & $\begin{array}{l}\text { In the absence of reliable evidence, it is the opinion of this work group that patients undergoing elective hip or knee arthroplasty, } \\
\text { and who also have a known bleeding disorder (e.g., hemophilia) and/or active liver disease, use mechanical compressive devices for } \\
\text { preventing venous thromboembolisme }\end{array}$ \\
\hline 8 & $\begin{array}{l}\text { In the absence of reliable evidence, it is the opinion of this work group that patients undergo early mobilization following elective } \\
\text { hip and knee arthroplasty. Early mobilization is of low cost, minimal risk to the patient, and consistent with current practice }\end{array}$ \\
\hline 9 & $\begin{array}{l}\text { Use of neuraxial (such as intrathecal, epidural, and spinal) anesthesia for patients undergoing elective hip or knee arthroplasty } \\
\text { to help limit blood loss, even though evidence suggests that neuraxial anesthesia does not affect the occurrence of venous } \\
\text { thromboembolic disease }\end{array}$ \\
\hline 10 & $\begin{array}{l}\text { Current evidence does not provide clear guidance about whether inferior vena cava (IVC) filters prevent pulmonary embolism } \\
\text { in patients undergoing elective hip and knee arthroplasty who also have a contraindication to chemoprophylaxis and/or known } \\
\text { residual venous thromboembolic disease. Therefore, we are unable to recommend for or against the use of such filters }{ }^{d}\end{array}$ \\
\hline
\end{tabular}

AAOS, American Academy of Orthopedic Surgeons

Reproduced after modification from AAOS clinical practice guidelines ${ }^{9,10}$

${ }^{a}$ A strong recommendation means that the benefits of the recommended approach clearly exceed the potential harm (or that the potential harm clearly exceeds the benefits in the case of a strong negative recommendation), and that the strength of the supporting evidence is high

${ }^{\mathrm{b}} \mathrm{A}$ moderate recommendation means that the benefits exceed the potential harm (or that the potential harm clearly exceeds the benefits in the case of a negative recommendation), but the strength of the supporting evidence is not as strong

'A limited recommendation means the quality of the supporting evidence that exists is unconvincing, or that well-conducted studies show little clear advantage to one approach vs another

${ }^{d}$ An inconclusive recommendation means that there is a lack of compelling evidence resulting in an unclear balance between benefits and potential harm ${ }^{\mathrm{e}} \mathrm{A}$ consensus recommendation means that expert opinion supports the guideline recommendation even though there is no available empirical evidence that meets the inclusion criteria of the guideline's systematic review

\section{Role of Prophylaxis}

The use of chemoprophylaxis in hospitalized trauma patients is based on unequivocal principles and scientific studies (Table 2). ${ }^{21-23}$ The conventional screening methods for detecting DVT have proven to be less effective in trauma population, making routine prophylaxis, a valuable tool in DVT prevention; however, the ideal mode and drug for prophylaxis, if any, are still not established ${ }^{24-27}$ (Table 3).

\section{Pelvis and Acetabulum Fractures}

These injuries are considered to be significant risk factors for development of VTE. ${ }^{1,28}$ Sen et al. in their study reported VTE in $29 \%$ of their patients undergoing surgeries for these fractures. ${ }^{1}$ Geerts et al. reported nonclinical VTE in $61 \%$ of their patients who were not given any chemoprophylaxis. ${ }^{29}$ These rates were much lower in studies by Steele et al. ${ }^{21}$ and Fishmann et al. ${ }^{22}$ who used prophylaxis and none of their patients developed clinical VTE.
Therefore, the role of chemoprophylaxis becomes important in this subset of trauma patients. In a review by Slobogean et al., LMWH was found to be efficient in decreasing the incidence of VTE in pelvi-acetabular fractures. ${ }^{30}$ The most recent ACCP guidelines also suggest chemoprophylaxis with $\mathrm{LMWH}$ in major trauma patients like these injuries. ${ }^{14}$ On the contrary, if the patient has any contraindications for $\mathrm{LMWH}$, at least mechanical prophylaxis needs to be given. El-Daly et al. ${ }^{31}$ recommend usage of heparin or fondaparinux for these injuries. In terms of period of prophylaxis, Sharma et al. ${ }^{32}$ recommend an extended period of up to 3 months. The Cochrane review for abdominal and pelvic surgeries also found that LMWH prophylaxis of up to 4 weeks after discharge is better than only indoor medication. ${ }^{33}$ Fondaparinux has been reported to be more or equally effective to $\mathrm{LMWH}$, but risk of bleeding is more. ${ }^{34}$ With no reversible agent and long half-life of 17 hours, bleeding risk becomes an important consideration for preferring LMWH over fondaparinux. ${ }^{34}$ Aspirin is another agent that can be used in 
Table 2: Rationale for thromboprophylaxis in hospitalized patients

\begin{tabular}{ll}
\hline Rationale & Description \\
\hline High prevalence of VTE & - DVT is common in many hospitalized patients \\
& - In hospital thrombus formation is usually clinically silent \\
& - At-risk patients unpredictably develop symptomatic thromboembolic compli- \\
& cations \\
& - Screening at-risk patients using physical examination or noninvasive testing is \\
& not very effective \\
Adverse consequences of unprevented & - Symptomatic DVT and/or fatal PE \\
VTE & - Increased costs of investigating and treating symptomatic patients \\
& - Risks in treatment like bleeding, increased risk of recurrent VTE, and/or post- \\
& thrombotic syndrome \\
Efficacy and effectiveness of thrombo- & Highly efficacious at preventing DVT and PE \\
prophylaxis & Cost-effectiveness of prophylaxis has repeatedly been demonstrated \\
\hline
\end{tabular}

Adapted from Geerts et al. ${ }^{29}$

VTE, venous thromboembolism; DVT, deep venous thrombosis; PE, pulmonary embolism

Table 3: List of commonly used anticoagulants

\begin{tabular}{|c|c|c|c|}
\hline \multirow[b]{2}{*}{ Agent } & \multicolumn{2}{|r|}{ Dosage } & \multirow[b]{2}{*}{ Special consideration } \\
\hline & DVT prophylaxis & DVT treatment & \\
\hline Unfractionated heparin & $\begin{array}{l}5000 \mathrm{USC} \\
\mathrm{BD} / \mathrm{TDS}\end{array}$ & $\begin{array}{l}80 \mathrm{U} / \mathrm{kg} \text { bolus followed } \\
\text { by } 18 \mathrm{U} / \mathrm{kg} / \mathrm{hour}\end{array}$ & $\begin{array}{l}\text { Renal failure: dose modification; Increased incidence of } \\
\text { thrombocytopenia }\end{array}$ \\
\hline Enoxaparin & $40 \mathrm{mg}$ sc OD & $1 \mathrm{mg} / \mathrm{kg} \mathrm{sc} \mathrm{BD}$ & In renal failure, dosage is halved \\
\hline Dalteparin & 5000 U sc OD & 5000 U sc BD & In renal failure, no dosage modification \\
\hline \multirow[t]{4}{*}{ Fondaparinux } & $2.5 \mathrm{mg} \mathrm{sc} \mathrm{OD}$ & Weight & $\begin{array}{l}\text { Contraindicated in patients with creatinine clearance } \\
<30 \mathrm{~mL} / \text { minute }\end{array}$ \\
\hline & & $<50$ kg: 5 mg sc daily & \\
\hline & & $\begin{array}{l}50-100 \mathrm{~kg}: 7.5 \mathrm{mg} \mathrm{sc} \\
\text { daily }\end{array}$ & \\
\hline & & >100 kg: 10 mg sc daily & \\
\hline Rivaroxaban & $20 \mathrm{mg}$ OD oral & $15 \mathrm{mg}$ oral $\mathrm{BD}$ & Contraindicated in patients with renal failure \\
\hline \multirow[t]{2}{*}{ Apixaban } & $2.5 \mathrm{mg} \mathrm{BD}$ oral & $\begin{array}{l}10 \mathrm{mg} \text { oral } \mathrm{BD} \text { for } 7 \text { days } \\
\text { and then } 5 \mathrm{mg} \text { oral } \mathrm{BD}\end{array}$ & Dose adjustment needed if any 2 of the following: \\
\hline & & & $\begin{array}{l}\text { Age }>80 \text { years; body weight }<60 \mathrm{~kg} \text {; serum } \\
\text { creatinine }>1.5 \mathrm{mg} / \mathrm{dL}\end{array}$ \\
\hline
\end{tabular}

major orthopedic surgeries, and its efficacy has been found to be at par with LMWH and fondaparinux by Kartikeyan et al. ${ }^{35}$ However, evidence still remains inconclusive in these fractures. Oral direct factor Xa inhibitors like apixaban and rivaroxaban can be given for up to 4 weeks postoperatively in pelvic-acetabular fractures as they have also been shown to decrease incidence of VTE. ${ }^{36}$ At our institute, we prefer apixaban for a postoperative period of 8 weeks, by which time patients are sufficiently mobilized.

\section{Hip Fractures}

Up to $7 \%$ of patients with hip fractures undergoing surgery develop fatal pulmonary embolism. ${ }^{37}$ Fractures of the femoral neck and intertrochanteric femur fractures are important risk factors of VTE. According to the ACCP, hip fracture patients must be given chemoprophylaxis that may be extended up to 5 weeks. ${ }^{14}$ Similarly, the Scottish intercollegiate guidelines network (SIGN) also recommends LMWH or fondaparinux for a period up to 4-6 weeks, and early mobilization and mechanical prophylaxis should be used as soon as possible. ${ }^{38}$ The SIGN guidelines do not recommend aspirin for the purpose, although the ACCP guidelines revised in 2012 consider aspirin as a potent modality. ${ }^{14}$ Marsland et al. ${ }^{39}$ state that aspirin does not provide protection to the extent provided by $\mathrm{LMWH}$. The literature recommends preoperative initiation of LMWH that may be stopped 12 hours prior to surgery and then restarted between 6 and 12 hours after surgery. ${ }^{14,40}$ Overall, routine prophylaxis is prevalent for fractures of hip and femur shaft; however, the efficacy of low-dose aspirin when compared to the other agents remains equivocal.

\section{Tibia Fractures}

In tibia fractures, the clots are generally distal as these rarely embolize proximally and prove fatal. ${ }^{41}$ Therefore, the guidelines for these fractures in terms of chemoprophylaxis is unclear. ${ }^{42}$ In a meta-analysis by Patterson and Morshed, LMWH was found to significantly reduce the incidence of VTE after tibia fractures treated operatively. ${ }^{43}$ However, in terms of clinically significant 
clots with symptoms or fatality, there was no significant efficacy. An important finding was that to prevent VTE in 1 patient, 31 patients had to be given a blanket prophylaxis. These figures became further exaggerated for clinically significant VTE, where 584 patients were given chemoprophylaxis to prevent actual clot in 1 patient. This is a very important point against routine chemoprophylaxis as the addition to the cost burden does not transform into clinical efficacy. However, the increased risk of bleeding due to chemoprophylaxis was not observed in this meta-analysis. ${ }^{43}$

The recommendations of Sagi et al. are that chemoprophylaxis should be reserved for patients of tibia fractures who additionally have other risk factors; in patients who do not have such added risk factors and can be mobilized after surgery, chemoprophylaxis should not be given. ${ }^{44}$ At our institute, we follow a similar protocol and do not use prophylaxis in patients who are mobile after surgery and do not have additional risk factors like obesity, diabetes, hypertension, elderly age group, etc. ${ }^{45}$

\section{Conclusion}

VTE is a common complication in orthopedic patients, and preventive strategies should be applied. In patients undergoing hip and knee arthroplasties or surgeries for pelvic-acetabular and hip fractures, routine postoperative chemoprophylaxis is a common protocol. LMWH or newer agents like apixaban or fondaparinux are effective in decreasing the incidence of clinical VTE and could be prescribed for a period as long as 6-8 weeks postoperatively. Patients with fractures distal to the knee do not require routine chemoprophylaxis unless they have other risk factors associated with VTE.

\section{References}

1. Sen RK, Kumar A, Tripathy SK, et al. Risk of postoperative venous thromboembolism in Indian patients sustaining pelvi-acetabular injury. Int Orthop 2011;35(7):1057-1063. DOI: 10.1007/s00264-0101093-6.

2. Agarwal S, Bhagwat AS, Modhe J. Deep vein thrombosis in Indian patients undergoing major lower limb surgery: Distribution of thrombi and its significance. Indian J Surg 2003;65:159-162. DOI: 10.1007/s13193-016-0538-9.

3. Dhillon KS, Askander A, Doraisamy S. Postoperative deep vein thrombosis in Asian patients is not a rarity. J Bone Joint Surg $\mathrm{Br}$ 1996;78:427-430. DOI: 10.1302/0301-620X.78B3.0780427.

4. Chan YK, Chiu KY. The incidence of DVT in elderly Chinese suffering hip fracture is low. J Orth Surg 2004;12(2):178-183. DOI: $10.1177 / 230949900401200208$.

5. Nagi ON, Dhillon MS, Katariya S, et al. Deep vein thrombosis after major surgery-evaluation by compression ultrasonography. Indian J Orthop 1999;33:200-203.

6. Sen RK, Tripathy SK, Singh AK. Is routine thromboprophylaxis justified among Indian patients sustaining major orthopedic trauma? A systematic review. Indian J Orthop 2011;45(3):197-207. DOI: 10.4103/0019-5413.80037.

7. Bagaria V, Modi N, Panghate A, et al. Incidence and risk factors for development of venous thromboembolism in Indian patients undergoing major orthopaedic surgery: Results of a prospective study. Postgrad Med J 2006;82:136-139. DOI: 10.1136/pgmj.2005. 034512.

8. Mavalankar AP, Majmundar D, Sudha R. Routine chemoprophylaxis for DVT in Indian patients. Indian J Orthop 2007;41:188-191. DOI: 10.4103/0019-5413.33680.

9. Lieberman JR, Pensak MJ. Prevention of venous thromboembolic disease after total hip and knee arthroplasty. J Bone Joint Surg Am 2013;95(19):1801-1811. DOI: 10.2106/JBJS.L.01328.
10. Mont MA, Jacobs JJ. AAOS clinical practice guideline: preventing venous thromboembolic disease in patients undergoing elective hip and knee arthroplasty. J Am Acad Orthop Surg 2011;19(12):777-778. DOI: 10.5435/00124635-201112000-00008.

11. Johanson NA, Lachiewicz PF, Lieberman JR, et al. American academy of orthopaedic surgeons clinical practice guideline on prevention of symptomatic pulmonary embolism in patients undergoing total hip or knee arthroplasty. J Bone Joint Surg Am 2009;91(7):1756-1757. DOI: 10.2106/JBJS.I.00511.

12. Eisenberg MJ, Richard PR, Libersan D, et al. Safety of short-term discontinuation of antiplatelet therapy in patients with drugeluting stents. Circulation 2009;119(12):1634-1642. DOI: 10.1161/ CIRCULATIONAHA.108.813667.

13. Geerts WH, Bergqvist D, Pineo GF, et al. American College of Chest Physicians. Prevention of venous thromboembolism:American College of Chest Physicians Evidence-Based Clinical Practice Guidelines (8th Edition). Chest 2008;133(6 Suppl):381S-453S. DOI: 10.1378/chest.08-0656.

14. Falck-Ytter Y, Francis CW, Johanson NA, et al. American College of Chest Physicians. Prevention of VTE in orthopedic surgery patients: Antithrombotic Therapy and Prevention of Thrombosis, 9th ed: American College of Chest Physicians Evidence-Based Clinical Practice Guidelines. Chest 2012;141(2 Suppl):e278S-e325S. DOI: 10.1378/chest.11-2404.

15. Pulmonary Embolism Prevention (PEP) Trial Col laborative Group. Prevention of pulmonary embolism and deep vein thrombosis with low dose aspirin: Pulmonary Embolism Prevention (PEP) trial. Lancet 2000;355(9212):1295-1302. DOI: 10.1016/S0140-6736(00)02110-3.

16. Miller RS, Weatherford DA, Stein D, et al. Antithrombin III and trauma patients: factors that determine low levels. J Trauma Inj Infect Crit Care 1994;37:442-445. DOI: 10.1097/00005373-199409000-00019.

17. Montgomery KD, Geerts WH, Code KI. Thromboembolic complications in patients with Pelvic trauma. Clin Orthop 1996;329:68-87. DOI: 10.1097/00003086-199608000-00010.

18. Selby R, Geerts W, Ofsosu FA, et al. Hypercoagulability after trauma: hemostatic charges and relationship to venous thromboembolism. Thromb Res 2009;124(3):281-287. DOI: 10.1016/j.thromres.2008.10.002.

19. Tubiana R, Duparc J. Prevention of Thromboembolic complications in orthopaedic and accidental injuries. J Bone Joint Surg (Br) 1961;43:7-51. DOI: 10.1302/0301-620X.43B1.7.

20. Stannard JP, Riley RS, McClenney MD, et al. Mechanical Prophylaxis against Deep-Vein Thrombosis After Pelvic and Acetabular Fractures. J Bone Joint Surg (Am) 2001;83:1047-1051. DOI: 10.2106/00004623200107000-00010.

21. Steele N, Dodenhoff RM, Ward AJ, et al. Thromboprophylaxis in pelvic and acetabular trauma surgery. The role of early treatment with lowmolecular-weight heparin. J Bone Joint Surg Br 2005;87:209-212. DOI: 10.1302/0301-620X.87B2.14447.

22. Fishmann AJ, Greeno RA, Brooks LR, et al. Prevention of deep vein thrombosis and pulmonary embolism in acetabular and pelvic fracture surgery. Clin Orthop Relat Res 1994;305:133-137. DOI: 10.1097/00003086-199408000-00017.

23. Velmahos GC. Posttraumatic thromboprophylaxis revisited: An argument against the current method of DVT and PE prophylaxis after injury. World J Surg 2006;30:483-487. DOI: 10.1007/s00268-005-0427-9.

24. Dennis J, Menawat WS, Von Thron J, et al. Efficacy of deep venous thrombosis prophylaxis in trauma patients and identification of high-risk groups. J Trauma 1993;35:132-138. DOI: 10.1097/00005373199307000-00021.

25. Burns GA, Cohn SM, Frumento RJ, et al. Prospective ultrasound evaluation of venous thrombosis in high risk trauma patients. JTrauma 1993;35:405-408. DOI: 10.1097/00005373-199309000-00012.

26. Comerota AJ, Katz ML, White JV. Why does prophylaxis with external pneumatic compression for deep vein thrombosis fail? Am J Surg 1992;164:265-268. DOI: 10.1016/S0002-9610(05)81083-9.

27. Ruiz AJ, Hill SL, Berry RE. Heparin, Deep Venous Thrombosis and trauma patients. Am J Surg 1991;162:159-162. DOI: 10.1016/00029610(91)90180-L. 
28. Sen RK, Kumar A, Tripathy S, et al. Risk factors of venous thromboembolism in Indian patients with pelvic-acetabular trauma. J Orthop Surg (Hong Kong) 2011 Apr;19(1):18-24. DOI: 10.1177/230949901101900105.

29. Geerts WH, Code KI, Jay RM, et al. A prospective study of venous thromboembolism after major trauma. N Engl J Med 1994;331: 1601-1606. DOI: 10.1056/NEJM199412153312401.

30. Slobogean GP, Lefaivre KA, Nicolaou S, et al. A systematic review of thromboprophylaxis for pelvic and acetabular fractures. J Orthop Trauma 2009;23(5):379-384. DOI: 10.1097/BOT.0b013e3181a5369c.

31. El-Daly I, Reidy J, Culpan P, et al. Thromboprophylaxis in patients with pelvic and acetabular fractures: a short review and recommendations. Injury 2013;44(12):1710-1720. DOI: 10.1016/j.injury.2013.04.030.

32. Sharma OP, Oswanski MF, Joseph RJ, et al. Venous thromboembolism in trauma patients. Am Surg 2007;73(11):1173-1180.

33. Rasmussen MS, Jørgensen LN, Wille-Jørgensen P. Prolonged thromboprophylaxis with low molecular weight heparin for abdominal or pelvic surgery. Cochrane Database Syst Rev 2009;(1):CD004318. DOI: 10.1002/14651858.CD004318.pub2.

34. de Alvarenga YR. New anticoagulants for venous thromboembolism prophylaxis in major orthopedic surgeries. A systematic review of randomized controlled trials. J Vasc Bras 2012;11:1-2. DOI: 10.1590/ S1677-54492012000100001.

35. Karthikeyan G, Eikelboom JW, Turpie AG, et al. Does acetyl salicylic acid (ASA) have a role in the prevention of venous thromboembolism? $\mathrm{Br} J$ Haematol 2009;146(2):142-149. DOI: 10.1111/j.1365-2141.2009.07734.x.

36. Monzon DG, Iserson KV, Cid A, et al. Oral thromboprophylaxis in pelvic trauma: a standardized protocol. J Emerg Med 2012;43(4):612-617. DOI: 10.1016/j.jemermed.2011.09.006.

37. Flevas DA, Megaloikonomos PD, Dimopoulos L, et al. Thromboembolism prophylaxis in orthopaedics: an update. EFORT
Open Rev 2018 Apr 27;3(4):136-148. DOI: 10.1302/2058-5241.3. 170018.

38. Scottish Intercollegiate Guidelines Network (SIGN). Prevention and management of venous thromboembolism. SIGN publication no. 122. Edinburgh: SIGN; 2010.

39. Marsland D, Mears SC, Kates SL. Venous thromboembolic prophylaxis for hip fractures. Osteoporos Int 2010;21(Suppl 4):S593-S604. DOI: 10.1007/s00198-010-1403-2.

40. Ktistakis I, Giannoudis V, Giannoudis PV. Anticoagulation therapy and proximal femoral fracture treatment: an update. EFORT Open Rev 2017;1:310-315. DOI: 10.1302/2058-5241.1.160034.

41. Selby R, Geerts WH, Crowther MA, et al. A prospective cohort study of the epidemiology of symptomatic venous thromboembolism (VTE) after isolated leg fractures distal to the knee without thromboprophylaxis. Blood 2004;104:143A. DOI: 10.1182/blood-200311-4085.

42. Cohen AT, Balaratnam S, Fassiadis N. Are isolated distal deep-vein thromboses clinically significant? Therapy 2008;5:151-157. DOI: 10.2217/14750708.5.2.151

43. Patterson JT, Morshed S. Chemoprophylaxis for Venous Thromboembolism in Operative Treatment of Fractures of the Tibia and Distal Bones: A Systematic Review and Meta-analysis. J Orthop Trauma 2017;31(9):453-460. DOI: 10.1097/BOT.0000000000000873.

44. Sagi HC, Ahn J, Ciesla D, et al. Venous thromboembolism prophylaxis 390 in orthopaedic trauma patients: a survey of OTA member practice patterns and OTA expert panel recommendations. J Orthop Trauma 2015;29:e355-e362. DOI: 10.1097/BOT.0000000000000387.

45. Cushman M. Epidemiology and risk factors for venous thrombosis. Semin Hematol 2007;44(2):62-69. DOI: 10.1053/ j.seminhematol.2007.02.004. 